\title{
PHONON PRECURSORS TO THE HIGH TEMPERATURE MARTENSITIC TRANSFORMATION IN Ti ${ }_{50} \mathrm{Pd}_{42} \mathrm{Cr}_{8}$
}

\author{
S. M. Shapiro, ${ }^{1}$ B. L. Winn, ${ }^{1}$ D. L. Schlagel, ${ }^{2}$ T. Lograsso, ${ }^{2}$ and R. Erwin ${ }^{3}$
}

1) Brookhaven National Laboratory, P.O. Box 5000, Upton, NY 11973-5000, USA

2) Ames Laboratory, Ames IA 50011, USA

3) National Institute of Standards and Technology (NIST), Gaithersburg, MD 20899, USA

\begin{abstract}
Inelastic neutron scattering measurements were carried out on the $\mathrm{Ti}_{50} \mathrm{Pd}_{50-\mathrm{x}} \mathrm{Cr}_{\mathrm{x}}$ alloy, which has the potential for being a high temperature shape memory material. For $\mathrm{x}=0$, the transformation temperature is $\sim 800 \mathrm{~K}$ and for the composition studied $\left(x=8\right.$ at.\%) $T_{M} \sim 400 \mathrm{~K}$. The majority of the measurements were performed in the parent, $\beta$-phase, up to $873 \mathrm{~K}$. Most of the phonons propagating along the three symmetry

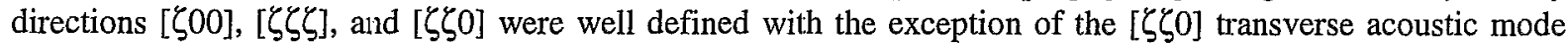
with displacements along the $[-\zeta \zeta 0]$ corresponding to the $C^{\prime}=1 / 2\left(C_{11}-C_{12}\right)$ elastic constant. These phonons are well defined for small $\zeta$, but for $\zeta>0.15$ they are strongly overdamped near the transition temperature, but become better defined at higher temperatures. An elastic peak develop in the cubic phase at $\zeta=0.22$ and increases in intensity as $T_{M}$ is approached. However, the dispersion curves show no anomaly at this particular wavevector, in marked contrast to the lattice dynamic studies of other systems exhibiting Martensitic transformations.
\end{abstract}

\section{INTRODUCTION}

Shape memory alloys (SMA) generate tremendous interest, in both, the scientific and technological area. The applications of SMA are nearly limitless and an excellent review was recently published [1]. However, most of the applications are within $\pm 100 \mathrm{C}$ of room temperature, since this corresponds to the transformation temperatures of most SMA. There is an interest in SMA with transition temperatures approaching $1000^{\circ} \mathrm{C}$ since it would open an entirely new area of applications in engine components. Three prime candidates for high temperature SMA are Ti-Pd, Nb-Ru, Ta-Ru alloys which have transformation temperatures near $800 \mathrm{~K}$ [2], $1400 \mathrm{~K}$ [3], and $1700 \mathrm{~K}$ [3], respectively. In this report we study the precursor phonon effects of the Martensitic transformation of a TiPd alloy by inelastic neutron scattering. A preliminary report has previously been published [4].

TiPd has been know to undergo displacive Martensitic transformation near $800 \mathrm{~K}$ from an ordered B2 (CsCl-type structure $\left(\mathrm{Pm} 3 \mathrm{~m}=: \mathrm{O}_{\mathrm{h}}{ }^{l}\right)$ to a $2 \mathrm{H}$ (B19) Martensite on cooling[2]. Upon alloying with various transition metals the transformation temperatures decreases and various intermediate incommensurate phases and different types of Martensites appear. The most extensive study of the phase diagram was by Mateeva[5] and reported by Enami [6]. Most of the work focused on the structure of the Martensitic phase, However a detailed study of TiPd-Cr investigated the modulated cubic structure that appears upon cooling by means of electron diffraction (ED) and high resolution transmission electron microscopy (HRTEM) for various Cr substitution [7].

\section{RESULTS AND DISCUSGIONS}

We chose to study the $\mathrm{Ti}_{50} \mathrm{Pd}_{42} \mathrm{Cr}_{8}$ alloy with a $\mathrm{Ms}$ temperature around $400 \mathrm{~K}$, because it is a more accessible temperature than the 50-50 alloy. The mosaic of the crystal in the cubic Austenite phase was better than $25^{\prime}$. The cubic lattice parameter was $3.162 \AA$ at $T=450 \mathrm{~K}$. The transformation temperatures were determined by carefully monitoring the intensity cubic [100] Bragg peak during a slow cooling and heating of the crystals: $M_{s}=407 \pm 5 \mathrm{~K}$ and $A_{f}=415 \pm 5 \mathrm{~K}$. The neutron experiments were performed at the NIST Center for Neutron Research using the BT9 triple axis spectrometer operating in the fixed Ef=14.7 $\mathrm{meV}$ mode. Pyrolytic graphite (PG) was used for monochromator and analyzer with a PG filter placed after the sample to eliminate higher order neutrons. The collimation chosen was $40^{\prime}-40^{\prime}-40^{\prime}-80^{\prime}$, which yielded an energy resolution of $\sim 1.0 \mathrm{meV}$. Most of the measurements were made in the $[\mathrm{H}, \mathrm{K}, 0]$ scattering plane centered around the $(1,2,0)$ Brillouin zone center. This is a very intense Bragg peak of the B2 structure because of $\mathrm{Ti}$ and $\mathrm{Pd}$ have opposite signs of their scattering lengths. 


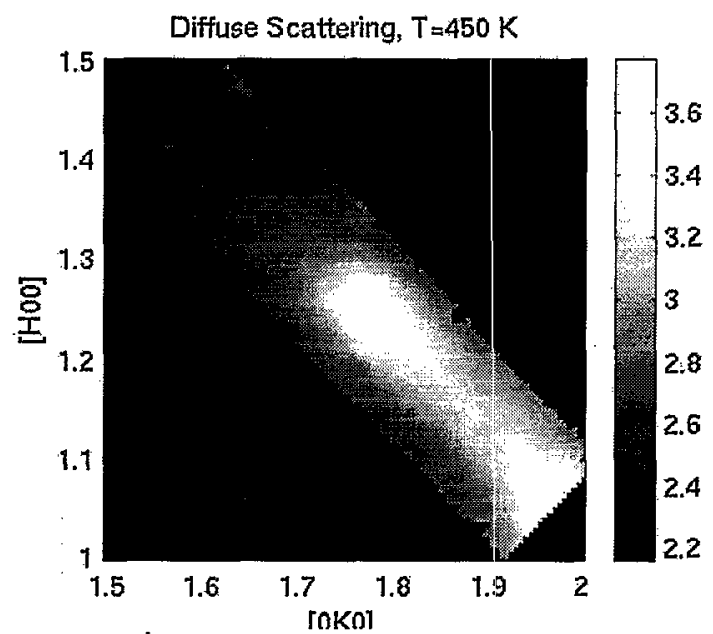

Figure 2. Intensity contours along the transverse direction at T-450K measured from the $(1,2,0)$ Brillouin zone center.

A scan along this direction from the $(1,2,0)$ zone center to the $(0,3,0)$ zone center is shown in Fig $3 a$
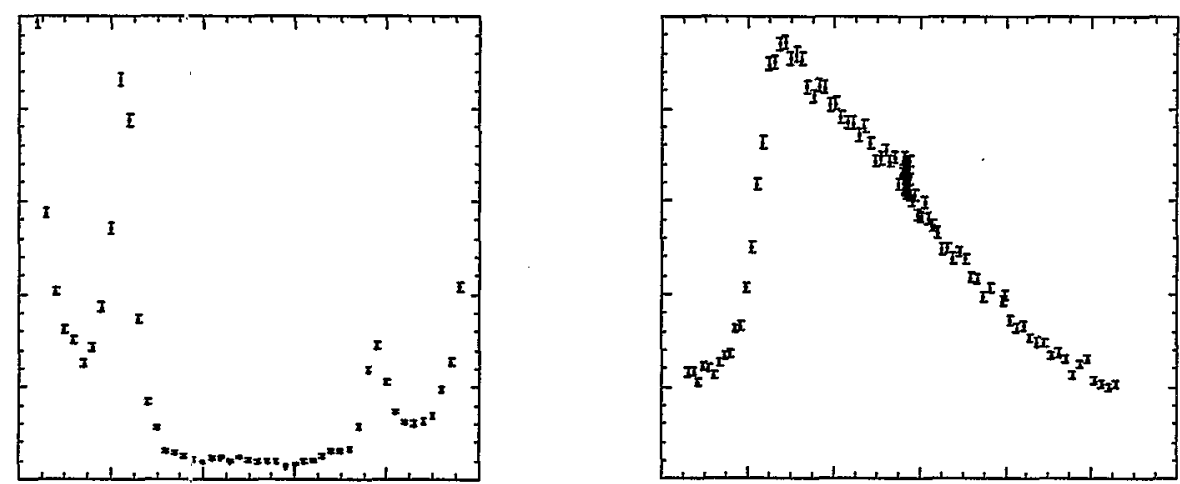

Figure 3. (a) An elastic scan measured from $(1,2,0)$ to $(0,3,0)$ at $T=450 \mathrm{~K}$. (b) Temperature dependence of $\mathrm{q}=0.22$ diffuse peak.

Two peaks are seen at the reduced value of $\zeta=(0.22,0.22,0)$ which implies that a structural modulation exists within the cubic phase. Fig $3 b$ shows the intensity of the peak as a function of temperature. It's position remains the same and the peak intensity increases upon cooling and exhibits a maximum at $\mathrm{M}_{\mathrm{s}}$, but is still present in the low temperature martensite phase but at a reduced level. The linewidth at high temperatures is nearly 5 times the instrumental resolution and it is nearly temperature independent. Its width would correspond to correlations along [110] of about 15 unit cells. The $\zeta=.22$ value of the modulation is smaller than $\zeta=.29$ measured in electron diffraction studies [7]. This discrepancy could be due to uncertainties in composition In this series of measurements on a single crystal we were unable to determine the low temperature structure, but it is reported to be B19 (2H) [2]

We also performed inelastic measurements of the TA2 branch at several temperatures. Fig 4 shows scans at two temperatures for two different $\zeta$ values. In Fig. $4 a, \zeta=0.1$, the peak is well defined at the highest temperature of the measurement, $\mathrm{T}=873 \mathrm{~K}$. As Ms is approached, at $\mathrm{T}=410 \mathrm{~K}$ the peak has broadened considerable and reduced its energy and a strong elastic peak developed. Figure $4 \mathrm{~b}$ shows the spectra along the same direction taken at $\zeta=0.25$. For the high temperature, the peak is very broad clearly shows a maximum. This indicates how quickly the broadening of the peak increases with $q$ which is also shown in Fig. 1 by the error bars on the TA2 branch. On cooling, a peak is no longer present and an intense elastic peak has developed that is shown in the contour plot of Fig. 2. A 


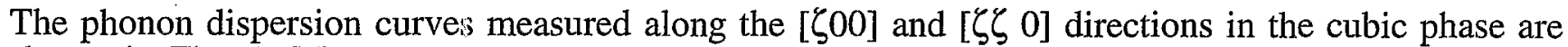
shown in Fig. 1. Most measurements were made at $\mathrm{T}=673 \mathrm{~K}$, except the TA2 mode, which was measured at $873 \mathrm{~K}$ The error bars shown for most of the curves are the estimates of the accuracy, except for the TA2 mode where the error bars represent the width of the phonon response.

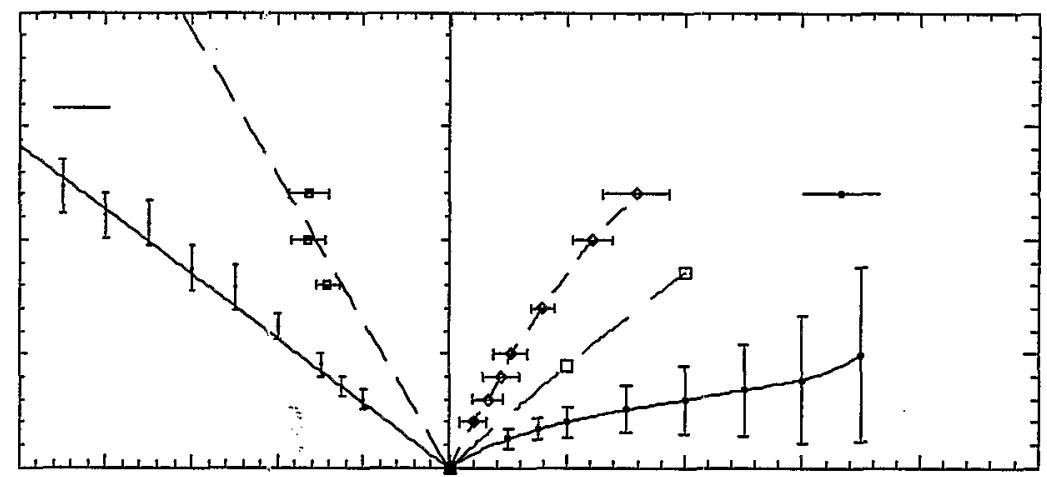

Figure 1: Measured dispersion curve along [00 $\left[\right.$ ] and $[\zeta \zeta 0]$ directions in $\mathrm{Ti}_{50} \mathrm{Pd}_{42} \mathrm{Cr}_{8}$. Except for the $\mathrm{TA} 2$ branch the measurements were performed at $673 \mathrm{~K}$. The error bars on TA2 correspond to the full-width-at-half maximum.

The slope of the dispersion curves for $\zeta \rightarrow 0$ determines the velocity of sound and, knowing the density, the elastic constants. In Table I we list the velocity of sound determined from the limiting slopes. From this

Table I: Velcoity of sound $\left(10^{4} \mathrm{~cm} / \mathrm{sec}\right)$ measured at $673 \mathrm{~K}$

\begin{tabular}{|l|l|l|l|}
\hline & LA & TA1 & TA2* \\
\hline$[001]$ & $49 \pm 5$ & $2.2 \pm 2$ & \\
\hline$[110]$ & $44 \pm 5$ & $23 . \pm 3$ & $12 \pm 3$ \\
\hline
\end{tabular}

*measured at $873 \mathrm{~K}$

we calculate the elastic constarits for $\mathrm{Ti}_{50} \mathrm{Pd}_{42} \mathrm{Cr}_{8}$, using the theoretical density of $7.64 \mathrm{gm} / \mathrm{cm}^{3}$;

$$
\begin{gathered}
\mathrm{C}_{11}=18.8 \times 10^{11} \mathrm{dynes} / \mathrm{cm}^{2} \\
\mathrm{C}_{44}=3.8 \times 10^{11} \mathrm{dynes} / \mathrm{cm}^{2} \\
\mathrm{C}_{12}=16.7 \times 10^{11} \text { dynes } / \mathrm{cm}^{2} \\
\mathrm{C}^{\prime}=1 / 2(\mathrm{C} 11-\mathrm{C} 12)=1.06 \times 10^{11} \text { dynes } / \mathrm{cm}^{2}
\end{gathered}
$$

The anomalous branch is the TA2 branch. In the limit of $\zeta 0$ this corresponds to C'. The atomic displacements associated with his mode correspond to a sliding of [110] planes along the perpendicular [1-10] direction. Zener showed long ago that this mode can lead to instability in the bcc-type lattice. Nearly allbcc-based martensites exhibit strong temperature dependent anomalies and diffuse scattering associated with this branch [8].

Let us now discuss the temperature dependent behavior of this transverse mode and the associated diffuse scattering. Figure 2 shows intensity contours of the elastic scattering measured along the transverse [110] direction from the $(1,2,0)$ Brillouin zone center for $M s<T=450 \mathrm{~K}$. There is a ridge of scattering with a pronounced peak at $\zeta=(0.22,0.22,0)$ plus diffuse scattering near $\zeta=0$ that is strongly temperature dependent. 

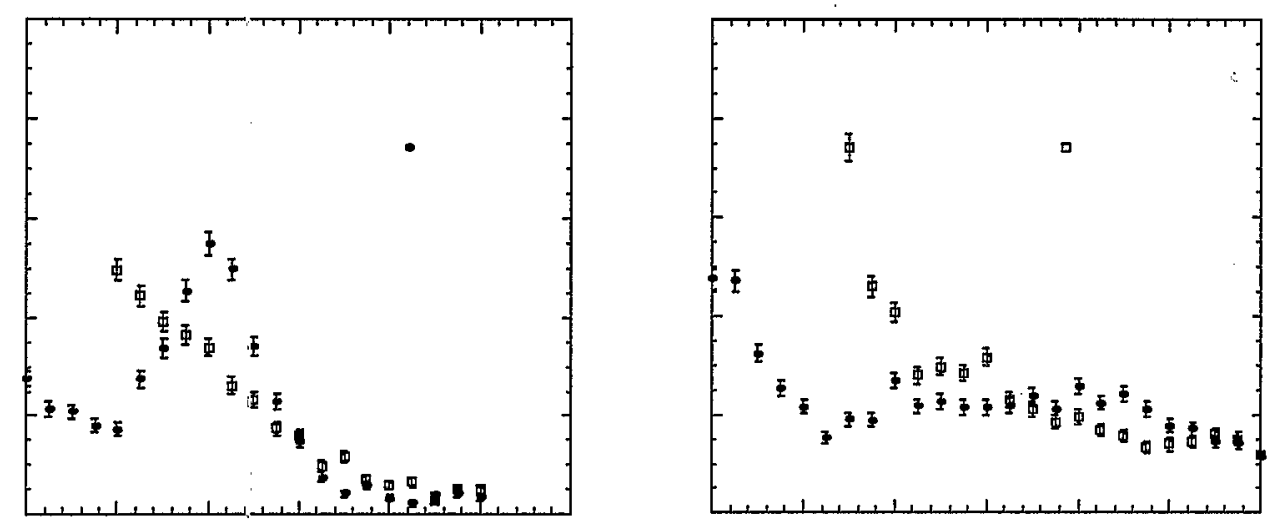

Firgure 4. Inelastic spectra measured at $\mathrm{Ms}<<\mathrm{T}=873 \mathrm{~K}$ and $\mathrm{Ms} \sim \mathrm{T}=410 \mathrm{~K}$ for [ $\zeta \zeta 0] \mathrm{TA} 2$ for (a) $\zeta=0.1$ and (b) $\zeta=0.25$.

surprising feature in this TiPd systems, when compared to other martensites, is that the phonon dispersion curve does not exhibit any pronounced anomaly at the same q-value as the appearance of the elastic diffuse peak. The line-broadening extends throughout the Brillouin zone.

In summary i)we deduced the elastic constants from the measurement of the phonon dispersion curves in $\mathrm{Ti}_{50} \mathrm{Pd}_{42} \mathrm{Cr}_{8}$, ii) measured a modulation of $\zeta=.22$ in the parent cubic phase and iii) observed an

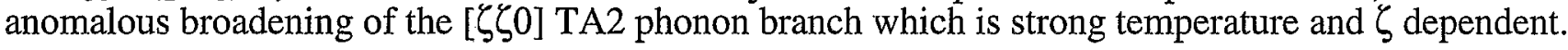

\section{Acknowledgements.}

The authors acknowledge the help of the technical staff at NIST during the course of this experiment. The work at Brookhaven is supported by the U. S. DOE under contract No. DE-AC02-98-CH10886 and the work at AMES under contract No. 7405 Eng.82.

\section{References}

[1] Science and Technology of Shape memory Alloys: New Developments, Otsuka, K. and Takeshita, T., Guest Editors, MRS Bulletin, 27, 91 (2002).

[2] Donkersloot, H. C. and van Vucht, J. H. N., J. Less Common Metals 20, 83 (1970).

[3] Fonda, R. W., Jones, H. N. and Vandemeer, R. A., Scripta met. 39, 1031 (1998).

[4] Winn, B. L., Shapiro, S. M., Erwin, R., Schlagel, D. L. and Lograsso, T.. Proceedings of International Conference on Neutron Scattering (ICNS-2001), To be published in Applied Physics A.

[5] Mateeva, N. M, Khachin, V. N. and V. P Sivokha: Stabil'nye i Metastabil'nye Faxovye

Ravnovesiya v Metallichesł.ikh Sistemakh. (Nauka Publ., Moskva), p.25 (1985).

[6] Enami, K and Nakagawa, $\$. Proceedings ICOMAT -92 (Monterey Inst. For Advanced Studies, Monterey, CA., 1993). P. 5\%1.

[7] Schwartz, A. J., Tanner, L. E. ibid, p. 95; Scripta Met. 32, 675 (1995).

[8] Shapiro, S. M., Yang, B. X. Noda, Y., Tanner, L. E., Schryvers, D., Phys. Rev. B 44, 9301 (1991). 